\title{
Nhận thức của sinh viên năm nhất chuyên ngành ngôn ngữ Anh về tầm quan trọng của việc tự học: Nghiên cứu tại Đại học Nông Lâm Thành phố Hồ Chí Minh
}

\section{First-year English majors' perceptions of the importance of learner autonomy: A case study at Nong Lam University}

\author{
Dương Mỹ Thẩm ${ }^{1 *}$, Nguyễn Đặng Thị Cúc Huyền ${ }^{1}$, Nguyễn Liên Hương ${ }^{1}$, \\ Hà Thị Ngọc Thương ${ }^{1}$ \\ ${ }^{1}$ Trường Đại học Nông Lâm Thành phố Hồ Chí Minh, Việt Nam \\ "Tác giả liên hệ, Email: tham.duongmy@hcmuaf.edu.vn
}

THÔNG TIN

DOI: $10.46223 / \mathrm{HCMCOUJS}$ soci.vi.15.1.597.2020

Ngày nhận: 23/03/2020

Ngày nhận lại: 23/04/2020

Duyệt đăng: 27/04/2020

Tù khóa:

nhận thức, sinh viên chuyên ngữ năm nhất, tự học

\section{TÓM TẮT}

Mục tiêu của nghiên cứu này là nhằm tìm hiểu nhận thức của sinh viên năm nhất chuyên ngành Ngôn Ngữ Anh, trường Đại học Nông Lâm Thành phố Hồ Chí Minh về tầm quan trọng của việc tự học đối với việc học của họ. Đây là một nghiên cứu định lượng với sự tham gia của 173 sinh viên. Họ được yêu cầu trả lời một bảng câu hỏi gồm 18 câu. Kết quả chỉ ra rằng hầu hết những sinh viên này đều công nhận vai trò quan trọng của việc tự học ở ba khía cạnh: khởi đầu, tổ chức-giám sát, đánh giá theo thứ tự giảm dần dựa vào giá trị trung bình. Cụ thể là họ nghĩ rằng khi gặp khúc mắc trong học tập thì họ truy cập internet, hỏi bạn bè và giáo viên; điều này giúp họ chủ động và tự tin hơn. Ngoài ra, họ cảm thấy có động lực học tập cao hơn khi tự chọn phương pháp học, chiến lược học, hoạt động luyện tập ngoài lớp học, tài liệu học tập và thực hiện kế hoạch học tập. Tuy nhiên, sinh viên vẫn chưa chắc chắn về hiệu quả các hoạt động tự đánh giá mang lại mặc dù họ tin rằng tự theo dõi tiến trình học tập và làm việc với bạn bè giúp họ xác định được điểm mạnh, điểm yếu của mình.

ABSTRACT
The study aimed at scrutinizing first-year English majors'
perceptions of the importance of learner autonomy toward their
English learning. This is quantitative research with the
participation of 173 students who answered an 18-item
questionnaire. The results based on mean scores indicated that the
participants recognized the considerable role of three aspects of
learner autonomy (i.e., initiating, organizing-monitoring \&
evaluating) in descending order. In particular, they thought that
they felt more proactive and confident when they viewed the




\section{Keywords:}

perception, first-year English major, learner autonomy

internet, peers and teachers as resources to deal with learning problems. In addition, their learning motivation increased as they chose to learn methods, learning strategies, out-of-class learning activities, learning materials and conducted a study plan. However, they showed slight hesitation in self-assessment and reflection although they believe that monitoring their learning process and working with peers can help them identify their own strengths and weaknesses.

\section{1. Đặt vấn đề}

Sự phát triển nhanh chóng của công nghệ dẫn đến những thay đổi đáng kể của hầu hết các mặt trong xã hội, đặc biệt là giáo dục. Những thiết bị công nghệ thật sự hỗ trợ cho quá trình học tập của người học. Họ có thể học bất kì nơi nào họ muốn thông qua việc sử dụng máy vi tính hay điện thoại. Littlemore (2001) đã chỉ ra ảnh hưởng tích cực của công nghệ đối với việc phát triển việc tự chủ trong học tập (gọi tắt là tự học). Trong bối cảnh việc đào tạo tiếng Anh trong thế kỷ XXI đang thay đổi thì khái niệm tự học được xem như là định hướng đúng đắn của quá trình học tập.

Sự khác biệt về văn hóa được xem là một trong những yếu tố ảnh hưởng đến việc tự học. Khái niệm tự học đã được nghiên cứu rộng rãi trên thế giới và được áp dụng vào thực tế giảng dạy mang lại nhiều lợi ích cho người học (Büyükdumana \& Şirin, 2010; Haseborg, 2012; Ismail \& Yusof, 2012; Macià, Ramos, Cervera \& Fuentes, 2003; Sanprasert, 2010). Tuy nhiên, một vài nghiên cứu chỉ ra sự khó khăn khi áp dụng vấn đề này ở các nước châu Á (Aliweh, 2011; Chau \& Cheng, 2010; Duong, 2015; Duong \& Seepho, 2014; Yunus \& Arshad, 2015). Tại Việt Nam, khái niệm tự học cũng dần được chú trọng phát triển trong những năm gần đây. Theo Nghị quyết số 29-NQ/TW Nghị quyết Hội nghị Trung ương 8 khóa XI về nội dung đổi mới căn bản, toàn diện giáo dục và đào tạo, đáp ứng yêu cầu công nghiệp hóa - hiện đại hóa trong điều kiện kinh tế thị trường định hướng xã hội chủ nghĩa và hội nhập quốc tế thì giáo dục đại học cần tập trung đào tạo nhân lực trình độ cao, bồi dưỡng nhân tài, phát triển phẩm chất và năng lực tự học, tự làm giàu tri thức, sáng tạo của người học. Trong đó, nội dung Nghị quyết nhấn mạnh năng lực tự học là yếu tố quyết định đến chất lượng học tập của sinh viên.

Mặc dù năng lực tự học được xem là kim chỉ nam cho hoạt động học tập của sinh viên trong thời đại mới nhưng vấn đề này chưa được quan tâm đúng mức trong giảng dạy và nghiên cứu, đặc biệt tại bối cảnh nghiên cứu. Theo quan sát của nhóm tác giả thì sinh viên năm nhất chuyên ngành Ngôn Ngữ Anh của Trường Đại học Nông Lâm Thành phố Hồ Chí Minh chưa được học các môn liên quan đến kỹ năng học đại học (bao gồm kỹ năng tự học). Vì vậy, việc khảo sát nhận thức của sinh viên năm nhất chuyên ngành Ngôn Ngữ Anh tại Đại học Nông Lâm Thành phố Hồ Chí Minh về tầm quan trọng của việc tự học và sau đó đưa ra những khuyến nghị giúp tăng cường năng lực tự học cho sinh viên là nhu cầu cấp thiết và có ý nghĩa quan trọng. Mục tiêu của nghiên cứu này là nhằm tìm hiểu nhận thức của sinh viên năm nhất chuyên ngành Ngôn Ngữ Anh tại Trường Đại học Nông Lâm Thành phố Hồ Chí Minh về tầm quan trọng của việc tự học với câu hỏi nghiên cứu sau: 
Sinh viên năm nhất chuyên ngành Ngôn ngữ Anh của Trường Đại học Nông Lâm Thành phố Hồ Chí Minh nhận thức như thế nào về tầm quan trọng của việc tự học?

\section{Cơ sở lý luận}

Holec (1981) được xem như là nhà nghiên cứu đầu tiên đưa ra định nghĩa cụ thể về khái niệm tự học. Theo ông, tự học là khả năng tự chịu trách nhiệm về việc học của mình. Định nghĩa này đóng vai trò định hướng cho các khái niệm về tự học sau này. Một thập kỷ sau, Little (1991) cũng cho rằng để trở thành người tự chủ trong học tập, người học trước tiên cần biết tự chịu trách nhiệm cho việc học của mình. Cotterall (2000) cụ thể hóa khái niệm trên bằng việc chỉ ra những hành động người học tự chủ cần làm, đó là phải xác định được mục tiêu học tập, nội dung và sự tiến bộ từng ngày, phải chọn phương pháp học phù hợp. Ngoài tính trách nhiệm, một số nhà nghiên cứu ở thế kỷ XX còn cho rằng khái niệm tự học liên quan đến tính độc lập (Dam, Eriksson, Little, Miliander \& Trebbi, 1990; Dickinson, 1987). Đến thế kỷ XXI, các nghiên cứu về vấn đề này cũng nhấn mạnh rằng người học cần phải có thái độ học tập độc lập và tương tác với bạn bè và giáo viên khi cần thiết, thậm chí không cần sự can thiệp của giáo viên (Little, 2009; Najeeb, 2013; Thanasoulas, 2000) và phải có khả năng đưa ra quyết định cho việc học của mình (Rivers \& Golonka, 2009; Scharle \& Szabó, 2000). Đáng kể nhất phải đề cập đến định nghĩa của Benson (2001) vì ông cho rằng thật thiếu sót nếu chỉ đặt khái niệm tự học trong mối quan hệ với tính trách nhiệm và tính tự quyết trong quá trình học. Theo ông, tự học được hiểu như là khả năng kiểm soát việc học của mình. Tóm lại, dù các nhà nghiên cứu xem xét khái niệm tự học ở góc độ nào thì tựu trung lại đều nhấn mạnh sự tự chủ của người học, gồm ba khía cạnh sau: Khởi đầu (initiating), tổ chức và giám sát (organizing \& monitoring) và đánh giá (evaluating). Có nghĩa là một người học tự chủ cần có khả năng tự chịu trách nhiệm, khả năng tự quyết và khả năng học tập độc lập.

Với sự thay đổi về phương pháp dạy và học, đặc biệt đối với việc học ngoại ngữ để đáp ứng những yêu cầu của một công dân toàn cầu trong thế kỷ XXI, năng lực tự học được xem như là chìa khóa quan trọng cho quá trình dạy và học hiệu quả. Chen và Pan (2015) cho rằng tự học là một cách tiếp cận mang tính thực tiễn cho việc dạy và việc học ngoại ngữ. Tương tự, Dang (2012) tin rằng năng lực tự học là mục tiêu cuối cùng của giáo dục nói chung và giáo dục ngoại ngữ nói riêng. Tác giả này giải thích rằng việc tăng cường năng lực tự học sẽ giúp tăng động lực học tập và tính chủ động trong quá trình học. Từ đó, họ có thái độ học tập tích cực. Bên cạnh đó, nhiều nhà nghiên cứu chỉ ra mối quan hệ tương hỗ của việc tự học và sự tương tác, học hỏi lẫn nhau giữa các sinh viên (Macaro, 1997; Reid, 1993; Stevens, 2007). Mỗi sinh viên có một phong cách học khác nhau; vì vậy, nếu họ làm việc với bạn bè sẽ giúp họ có cơ hội học hỏi lẫn nhau (Blidi, 2017). Một người có năng lực tự học có thể làm việc một mình, theo cặp hoặc theo nhóm tùy vào nhu cầu của họ. Ngoài sự tương tác với bạn bè, người tự học có thể đặt mục tiêu học tập, xây dựng kế hoạch học tập, chọn phương pháp học và chiến lược học phù hợp, chọn tài liệu học tập, tự đánh giá việc học của mình, chủ động tìm kiếm sự giúp đỡ khi cần thiết (Ganza, 2008; Gardner \& Miller, 1999; Littlemore, 2001; Wenden, 1991). Theo Little (1991), người học có thể tự xây dựng một chương trình học tập cho riêng mình nếu họ được trao quyền tự quyết. Và khi họ có thể tự quyết về việc học của mình thì sẽ giúp tăng tính trách nhiệm. Điều này cho thấy rằng nếu người học có cơ hội tự đưa ra những quyết định, tự chịu trách nhiệm cho việc học và học tập độc lập không cần sự can thiệp nhiều của giáo viên 
nếu được hướng dẫn đúng cách. Việc tự học còn ảnh hưởng tích cực đến kết quả học tập như một vài nghiên cứu đã đưa ra. Một khi tính trách nhiệm đối với việc học được nâng cao thì kéo theo kết quả học tập sẽ được cải thiện (Crabbe, 1993). Little (2009) cũng cho rằng khi người học tự đánh giá được việc học của mình thì họ có xu hướng tập trung vào việc học hơn, điều này góp phần vào kết quả học tập tốt.

Nói chung, năng lực tự học đóng vai trò cốt lõi trong giáo dục thời đại mới. Việc học ngoại ngữ là một quá trình lâu dài đòi hỏi nhiều nỗ lực của cả người dạy và người học (Chen \& Pan, 2015). Vì vậy, họ cần được cung cấp kiến thức và kỹ năng đầy đủ về việc tự học.

\section{Phương pháp nghiên cứu}

\subsection{Bối cảnh nghiên cúu}

Nghiên cứu này được thực hiện tại Khoa Ngoại Ngữ - Sư Phạm (tiền thân là Khoa Ngoại Ngữ), Trường Đại học Nông Lâm Thành phố Hồ Chí Minh. Số lượng sinh viên hiện tại của Khoa gồm 856 sinh viên; trong đó có 193 sinh viên chuyên ngành tiếng Anh năm nhất. Về đội ngũ giảng viên, Khoa Ngoại Ngữ - Sư Phạm hiện có 24 giảng viên cơ hữu được phân công công tác tại 05 bộ môn gồm Ngôn ngữ - Văn hóa - Văn chương, Tiếng Anh Quản Lý và Không Chuyên, Sư Phạm Tiếng Anh, Tiếng Pháp, và Sư Phạm Kỹ Thuật.

Từ ngày 05/9/2008, Trường Đại học Nông Lâm Thành phố Hồ Chí Minh áp dụng hệ thống tín chỉ thay cho hệ thống niên chế. Tín chỉ được sử dụng để tính khối lượng học tập của sinh viên. Một tín chỉ được quy định bằng 15 tiết học lý thuyết. Theo quy định của Nhà trường, sinh viên năm nhất chuyên ngành tiếng Anh cần tích lũy tối đa 30 tín chỉ và phải đạt điểm trung bình chung tích lũy từ 2,00 trở lên. Dựa vào bảng phân loại giờ tín chỉ như trên thì sinh viên cần phải bố trí thời gian và có phương pháp tự học hợp lý.

\section{2. Đối tựng khảo sát}

Đối tượng khảo sát của nghiên cứu này gồm tất cả sinh viên năm nhất chuyên ngành Ngôn Ngữ Anh của Trường Đại học Nông Lâm Thành phố Hồ Chí Minh (trừ 3 bạn tham gia nghiên cứu thử nghiệm). Tuy nhiên, chúng tôi chỉ ghi nhận 173/190 phiếu hợp lệ. Vì vậy, đối tượng khảo sát gồm 141 nữ (chiếm 80,5\%) và 32 nam (chiếm 18,5\%). Về khoảng thời gian đã học tiếng Anh, có 76 sinh viên học tiếng Anh từ 7-10 năm (chiếm 43,9\%), 57 sinh viên học tiếng Anh dưới 7 năm (chiếm 32,9\%) và 40 sinh viên đã học tiếng Anh trên 10 năm (chiếm 23,1\%). Đối với hình thức tự học họ yêu thích, 92 sinh viên chọn học một mình (chiếm 53,2\%), 47 sinh viên chọn học theo nhóm (chiếm 27,2\%) và 34 sinh viên chọn học theo cặp (chiếm $19,7,9 \%)$.

\subsection{Phuơng pháp thu thập và phân tích dĩ liệu}

Bảng khảo sát được xây dựng nhằm mục đích tìm hiểu nhận thức của sinh viên năm nhất chuyên ngành tiếng Anh của Trường Đại học Nông Lâm Thành phố Hồ Chí Minh về tầm quan trọng của việc tự học. Bảng câu hỏi này được xây dựng dựa trên cơ sở lý thuyết về tầm quan trọng của việc tự học (Blidi, 2017; Crabbe, 1993; Ganza, 2008; Gardner \& Miller, 1999; Littlemore, 2001; Rubin \& Thompson, 1994; Scharle \& Szabó, 2000; Wenden, 1991) và đồng thời kế thừa từ bảng câu hỏi trong nghiên cứu của Liu (2012). Bảng câu hỏi sử dụng tiếng Việt 
để không gây khó khăn về mặt ngôn ngữ cho đối tượng khảo sát, gồm hai phần: (1) thông tin cá nhân dùng để thu thập dữ liệu về giới tính, khoảng thời gian đã học tiếng Anh, hình thức tự học yêu thích và (2) tầm quan trọng của việc tự học trong quá trình học tiếng Anh gồm 18 câu hỏi với hệ số tin cậy (Cronbach's Alpha) là 0,84 . Theo bảng phân loại của George và Mallery (2003) thì hệ số tin cậy của bảng khảo sát này nằm trong khoảng tốt $(0,80<\alpha<0,90)$. Điều này có nghĩa là bảng khảo sát đủ tin cậy để thu thập dữ liệu cho nghiên cứu này. Ngoài ra, để đảm bảo độ tin cậy của công cụ thu thập dữ liệu, nhóm nghiên cứu phát bảng câu hỏi cho 03 sinh viên năm nhất tại bối cảnh nghiên cứu (không tham gia nghiên cứu chính) trả lời thử để xem nội dung và hình thức của bảng câu hỏi có gây cản trở gì cho đối tượng khảo sát không. Sau đó chúng tôi điều chỉnh lại bảng câu hỏi cho phù hợp.

Để phân tích dữ liệu định lượng được thu thập từ bảng câu hỏi, nhóm tác giả dùng thống kê mô tả thông qua phần mềm SPSS phiên bản 20 , gồm giá trị trung bình $(\mathrm{M})$ và độ lệch chuẩn (SD). Giá trị trung bình theo thang đo 5 mức độ của Likert được quy đổi thành các khoảng dưới đây:

1,00 - 1,80: Rất không đồng ý

1,81 - 2,60: Không đồng ý

2,61 - 3,40: Bình thường

3,41 - 4,20: Đồng ý

4,21 - 5,00: Rất đồng ý

\section{Kết quả và thảo luận}

\subsection{Kết quả}

Phần này trình bày kết quả về nhận thức của sinh viên năm nhất chuyên ngành Ngôn Ngữ Anh của Đại học Nông Lâm Thành phố Hồ Chí Minh về tầm quan trọng của việc tự học trong quá trình học của họ. Việc tự học được xét ở ba khía cạnh như sau: (1) khởi đầu, (2) tổ chức- giám sát, (3) đánh giá. Nội dung của Bảng 1 là về giá trị trung bình tổng của ba nhóm. Kết quả cho thấy nhóm khởi đầu có giá trị trung bình cao nhất $(\mathrm{M}=4,09 ; \mathrm{SD}=0,57)$, tiếp đến là nhóm tổ chức-giám sát với giá trị trung bình tương đối cao $(\mathrm{M}=3,95 ; \mathrm{SD}=0,53)$, và giá trị trung bình thấp nhất thuộc về nhóm đánh giá $(\mathrm{M}=3,58 ; \mathrm{SD}=0,49)$. Điều này có nghĩa là hầu hết sinh viên đồng tình với các ý kiến trong ba nhóm này theo thứ tự từ cao đến thấp.

\section{Bảng 1}

Giá trị trung bình tổng của ba khía cạnh tự học

\begin{tabular}{clccc}
\hline \multirow{2}{*}{ STT } & \multirow{2}{*}{ Nội dung } & \multirow{2}{*}{ Hạng } & \multicolumn{2}{c}{$\mathbf{n = 1 7 3}$} \\
\cline { 4 - 5 } & Khởi đầu & 1 & 4,09 & SD \\
\hline 2 & Tổ chúcc-giám sát & 2 & 3,95 & 0,57 \\
\hline 3 & Đánh giá & 3 & 3,58 & 0,53 \\
\hline
\end{tabular}

Ghi chú: n: số lượng mẫu, M: Giá trị trung bình, SD: Độ lệch chuẩn

Nguồn: Kết quả phân tích dữ liệu của nhóm nghiên cứu 
Dưới đây là phần báo cáo kết quả giá trị trung bình của từng mục trong ba nhóm kể trên. Theo quan sát từ Bảng 2 về giá trị trung bình của nhóm khởi đầu, sinh viên cho rằng việc tự học giúp họ nhiều nhất khi họ cảm thấy chủ động hơn vì tự giải đáp thắc mắc với sự hỗ trợ của internet $(\mathrm{M}=4,51 ; \mathrm{SD}=0,65)$. Việc trao đổi với bạn bè những vấn đề chưa hiểu cũng giúp họ cảm thấy tự tin hơn; Vì thế, họ khá đồng tình với ý kiến này $(\mathrm{M}=4,05 ; \mathrm{SD}=0,94)$. Ngoài ra, họ còn cho rằng việc tự học giúp cho họ theo dõi được tiến trình học của mình, cụ thể là biết được nên học gì tiếp theo dựa vào mục tiêu đã thiết lập trước đó $(\mathrm{M}=3,94 ; \mathrm{SD}=0,80)$. Cuối cùng, khi gặp vấn đề không hiểu họ chủ động tìm gặp giáo viên nhờ giúp đỡ là lợi ích mà khá nhiều sinh viên cho rằng do việc tự học mang lại $(\mathrm{M}=3,85 ; \mathrm{SD}=0,99)$.

\section{Bảng 2}

Giá trị trung bình của nhóm khởi đầu

\begin{tabular}{|c|c|c|c|c|}
\hline \multirow{2}{*}{ Câu } & \multirow{2}{*}{ Việc tự học giúp tôi... } & \multirow{2}{*}{ Hạng } & \multicolumn{2}{|c|}{$n=173$} \\
\hline & & & $\mathbf{M}$ & SD \\
\hline 1 & $\begin{array}{l}\text { biết được mình nên học gì tiếp theo khi xác định mục } \\
\text { tiêu học tập của mình. }\end{array}$ & 3 & 3,94 & 0,80 \\
\hline 2 & $\begin{array}{l}\text { chủ động hơn khi hỏi giáo viên một số vấn đề không } \\
\text { hiểu. }\end{array}$ & 4 & 3,85 & 0,99 \\
\hline 3 & tự tin hơn khi hỏi bạn bè một số vấn đề không hiểu. & 2 & 4,05 & 0,94 \\
\hline 4 & $\begin{array}{l}\text { chủ động hơn khi truy cập internet để giải đáp thắc } \\
\text { mắc. }\end{array}$ & 1 & 4,51 & 0,65 \\
\hline
\end{tabular}

Ghi chú: n: số lượng mẫu, M: Giá trị trung bình, SD: Độ lệch chuẩn

Nguồn: Kết quả phân tích dữ liệu của nhóm nghiên cứu

Theo số liệu trong Bảng 3 về giá trị trung bình của nhóm tổ chức-giám sát, sinh viên tương đối đồng tình với tất cả các ý kiến liên quan đến quá trình tổ chức-giám sát. Cụ thể là số đông sinh viên cảm thấy hứng thú hơn khi họ được chọn các hoạt động ngoài lớp như: nghe các bài hát bằng tiếng $\mathrm{Anh}(\mathrm{M}=4,26 ; \mathrm{SD}=0,80)$, xem phim bằng tiếng $\mathrm{Anh}(\mathrm{M}=4,26 ; \mathrm{SD}=0,69)$ và luyện nói với bạn bè $(\mathrm{M}=3,98 ; \mathrm{SD}=0,80)$. Do vậy, giá trị trung bình của những ý kiến này là cao nhất nhóm. Tuy nhiên, cùng là các hoạt động ngoài giờ học nhưng ít sinh viên đồng tình hơn với ý kiến cho rằng đọc báo, tạp chí bằng tiếng Anh ngoài lớp giúp họ hứng thú hơn. Vì thế, giá trị trung bình của ý kiến này được xếp ở vị trí khá thấp trong nhóm $(\mathrm{M}=3,84 ; \mathrm{SD}=0,84)$.

Nhóm các ý kiến có giá trị trung bình cao thứ hai liên quan đến xác định phương pháp học phù hợp $(\mathrm{M}=3,98 ; \mathrm{SD}=0,90)$, xây dựng và thực hiện kế hoạch học tập $(\mathrm{M}=3,91 ; \mathrm{SD}=0,83)$, xác định chiến lược học $(\mathrm{M}=3,91 ; \mathrm{SD}=0,92)$ và tự chọn các hoạt động học $(\mathrm{M}=3,88 ; \mathrm{SD}=0,92)$. Điều này có nghĩa là nhiều sinh viên cho rằng việc tự học giúp cho họ có động lực học tập và kiểm soát được việc học của mình. So với các khía cạnh tự học kể trên thì việc tự chọn tài liệu 
học kể cả trong lớp học $(\mathrm{M}=3,83 ; \mathrm{SD}=0,81)$ và ngoài lớp học $(\mathrm{M}=3,66 ; \mathrm{SD}=0,88)$ dường như ít giúp họ có động lực học tập hơn với các giá trị trung bình thấp nhất nhóm.

\section{Bảng 3}

Giá trị trung bình của nhóm tổ chức-giám sát

\begin{tabular}{|c|c|c|c|c|}
\hline \multirow{2}{*}{ Câu } & \multirow{2}{*}{ Việc tự học giúp tôi... } & \multirow{2}{*}{ Hạng } & \multicolumn{2}{|c|}{$\mathrm{n}=\mathbf{1 7 3}$} \\
\hline & & & $\mathbf{M}$ & SD \\
\hline 5 & $\begin{array}{l}\text { biết được mình nên học gì tiếp theo khi xây dựng và } \\
\text { thực hiện kế hoạch học tập. }\end{array}$ & 5 & 3,92 & 0,84 \\
\hline 6 & $\begin{array}{l}\text { hứng thú hơn khi tự chọn các hoạt động học trong } \\
\text { các bài học tiếng Anh. }\end{array}$ & 7 & 3,88 & 0,92 \\
\hline 7 & $\begin{array}{l}\text { có động lực học tập cao hơn khi xác định được } \\
\text { phương pháp học phù hợp. }\end{array}$ & 4 & 3,98 & 0,90 \\
\hline 8 & $\begin{array}{l}\text { có động lực học tập hơn khi xác định chiến lược học } \\
\text { phù hợp. }\end{array}$ & 6 & 3,91 & 0,92 \\
\hline 9 & $\begin{array}{l}\text { hứng thú hơn khi chọn hoạt động nghe bài hát bằng } \\
\text { tiếng Anh ngoài lớp học. }\end{array}$ & 1 & 4,26 & 0,80 \\
\hline 10 & $\begin{array}{l}\text { hứng thú hơn khi chọn hoạt động đọc báo/tạp chí } \\
\text { bằng tiếng Anh ngoài lớp học. }\end{array}$ & 8 & 3,84 & 0,84 \\
\hline 11 & $\begin{array}{l}\text { hứng thú hơn khi chọn hoạt động luyện nói tiếng } \\
\text { Anh với bạn bè ngoài lớp học. }\end{array}$ & 3 & 3,98 & 0,80 \\
\hline 12 & $\begin{array}{l}\text { hứng thú hơn khi chọn hoạt động xem phim bằng } \\
\text { tiếng Anh ngoài lớp học. }\end{array}$ & 2 & 4,26 & 0,69 \\
\hline 13 & $\begin{array}{l}\text { có động lực học tập hơn khi tự chọn tài liệu học trong } \\
\text { lớp học. }\end{array}$ & 9 & 3,83 & 0,81 \\
\hline 14 & $\begin{array}{l}\text { có động lực học tập hơn khi tự chọn tài liệu học } \\
\text { ngoâi lớp học. }\end{array}$ & 10 & 3,66 & 0,88 \\
\hline
\end{tabular}

Ghi chú: n: số lượng mẫu, M: Giá trị trung bình, SD: Độ lệch chuẩn

Nguồn: Kết quả phân tích dữ liệu của nhóm nghiên cứu

Theo kết quả ở Bảng 1, nhóm đánh giá có giá trị trung bình tổng thấp nhất trong ba nhóm. Điều này có nghĩa là sinh viên ít đồng tình với các ý kiến trong nhóm này hơn hai nhóm còn lại. Thật vậy, nhìn vào Bảng 4 ta thấy chỉ có hai ý kiến liên quan đến việc học tập với bạn bè giúp họ xác định được điểm mạnh, điểm yếu của chính mình $(\mathrm{M}=3,89 ; \mathrm{SD}=0,86)$ và việc theo dõi tiến trình học tập giúp sinh viên tự tin hơn $(\mathrm{M}=3,83 ; \mathrm{SD}=0,74)$ có giá trị trung bình khá cao. Nói cách khác, sinh viên hầu như đồng tình với hai ý kiến này. Tuy nhiên, họ lại không nghĩ rằng việc tự đánh giá năng lực của chính mình giúp họ đạt kết quả học tập cao hơn $(\mathrm{M}=3,30 ; \mathrm{SD}=0,79)$ và việc viết nhật ký hoặc suy ngẫm lại việc học giúp họ kiểm soát việc học tốt hơn $(\mathrm{M}=3,29 ; \mathrm{SD}=0,88)$. 


\section{Bảng 4}

Giá trị trung bình của nhóm đánh giá

\begin{tabular}{|c|c|c|c|c|}
\hline \multirow{2}{*}{ Câu } & \multirow{2}{*}{ Việc tự học giúp tôi... } & \multirow{2}{*}{ Hạng } & \multicolumn{2}{|c|}{$n=173$} \\
\hline & & & $\mathbf{M}$ & SD \\
\hline 15 & $\begin{array}{l}\text { tự tin hơn khi nhận thấy sự tiến bộ của mình qua các } \\
\text { bài học thông qua việc theo dõi tiến trình học tập của } \\
\text { mình. }\end{array}$ & 2 & 3,83 & 0,74 \\
\hline 16 & $\begin{array}{l}\text { xác định được điểm mạnh, điểm yếu của mình khi } \\
\text { làm việc với bạn bè. }\end{array}$ & 1 & 3,89 & 0,86 \\
\hline 17 & $\begin{array}{l}\text { có kết quả học tập cao hơn khi tôi có thể tự đánh giá } \\
\text { năng lực của mình. }\end{array}$ & 3 & 3,29 & 0,88 \\
\hline 18 & $\begin{array}{l}\text { kiểm soát được việc học tốt hơn thông qua việc viết } \\
\text { nhật ký học tập hoặc việc suy ngẫm lại mình đã học } \\
\text { được gì. }\end{array}$ & 4 & 3,30 & 0,79 \\
\hline
\end{tabular}

Ghi chú: n: số lượng mẫu, M: Giá trị trung bình, SD: Độ lệch chuẩn

Nguồn: Kết quả phân tích dữ liệu của nhóm nghiên cứu

\subsection{Thảo luận}

Tầm quan trọng của việc tự học được đề cập ở ba khía cạnh: khởi đầu, tổ chức-giám sát và đánh giá. Phần thảo luận kết quả phân tích được trình bày dựa vào ba khía cạnh kể trên.

Xét về nhóm khởi đầu, internet được xem là một nguồn học liệu hữu ích khi sinh viên gặp các vấn đề khó hiểu vì họ cho rằng việc làm này giúp họ chủ động hơn trong học tập. Có rất nhiều nhà nghiên cứu đã chỉ ra tầm ảnh hưởng của khoa học công nghệ với sự hỗ trợ của internet đối với việc học nói chung và học ngôn ngữ nói riêng (Kurtz, 2012; Littlemore, 2001; Noytim, 2006). Ngoài ra, bạn bè và giáo viên cũng là nguồn nhân lực đáng tin cậy họ cần khai thác để giúp họ học tập chủ động hơn (Blidi, 2017; Little, 2004; Voller, 1997).

Xét về nhóm tổ chức-giám sát, sinh viên tin rằng họ sẽ hứng thú hơn khi tự chọn hoạt động ngoài lớp học mang tính vừa học vừa chơi như nghe nhạc, xem phim, đọc báo/tạp chí hoặc nói chuyện với bạn bằng tiếng Anh. Ngoài ra, họ còn có động lực học hơn khi xây dựng và thực hiện kế hoạch học tập, chọn phương pháp học, xác định chiến lược học tập, và chọn hoạt động học trong các bài học như nhiều nhà nghiên cứu đã khẳng định (Ganza, 2008; Gardner \& Miller, 1999; Littlemore, 2001; Rubin \& Thompson, 1994; Scharle \& Szabó, 2000; Wenden, 1991). Riêng ý kiến cho rằng sinh viên có động lực học tập hơn khi tự chọn tài liệu trong và ngoài lớp học nhận được ít sự đồng thuận nhất từ các đối tượng khảo sát. Điều này có thể là do sinh viên tại bối cảnh nghiên cứu chưa có nhiều cơ hội, thậm chí là không có cơ hội chọn tài liệu học cho mình mà họ phải thực hiện theo chương trình đào tạo dưới sự hướng dẫn của giáo 
viên. Mặc dù vậy, họ vẫn tin rằng hoạt động này giúp tăng động lực học cho họ. Nhiều kết quả của các nghiên cứu trước đây cũng đưa ra kết quả tương tự (Littlewood, 1997; Nunan, 1996, 1997; Rivers \& Golonka, 2009).

Xét về nhóm đánh giá, sinh viên cho rằng việc họ tự đánh giá sự tiến bộ của mình giúp họ tự tin hơn cũng như xác định được điểm mạnh, điểm yếu của chính mình khi làm việc với bạn bè. Khi được hỏi về phương thức tự học yêu thích, gần một nửa đối tượng khảo sát (46.8\%) chọn học theo cặp và nhóm. Sự tương tác với bạn bè trong học tập là một một định hướng đúng đắn trong bối cảnh dạy và học ở thế kỷ XXI. Khi làm việc với nhau, người học sẽ nâng cao tính trách nhiệm và có điều kiện học hỏi lẫn nhau (Blidi, 2017; Macaro, 1997; Reid, 1993; Stevens, 2007). Tuy nhiên, họ lại có phần nghi ngờ về việc tự đánh giá năng lực giúp họ có kết quả học tập cao hơn và việc suy ngẫm lại việc học (viết nhật ký) giúp họ kiểm soát việc học tốt hơn. Điều này có thể suy ra rằng có lẽ họ đã gặp vấn đề nào đó gây cản trở cho việc áp dụng hai hoạt động này trong quá trình học. Do đó, sinh viên cần được hướng dẫn cách làm cụ thể và cần sự tư vấn của giáo viên khi gặp vấn đề khó khăn trong suốt quá trình học. Trong một nghiên cứu của mình, Scheb-Buenner (2018) đã đưa ra kết luận rằng việc theo dõi và đánh giá quá trình học nên là nhiệm vụ của cả giáo viên và sinh viên và sự hướng dẫn cụ thể là cần thiết cho sinh viên để mang lại hiệu quả cao nhất. Duong (2015) cũng chỉ ra rằng sinh viên không tự tin với kiến thức và kinh nghiệm của mình khi tự đánh giá bài viết của mình hay đánh giá bài viết của bạn cùng lớp. Họ cần sự đánh giá của cả giáo viên để đảm bảo chất lượng của bài viết.

\section{Kết luận và khuyến nghị}

\subsection{Kết luận}

Nhìn chung, đa số đối tượng khảo sát đồng tình với các ý kiến về tầm quan trọng của việc tự học đối với việc học của họ. Trong ba khía cạnh tự học thì các ý kiến liên quan đến khía cạnh đánh giá nhận được ít sự đồng tình hơn các ý kiến ở hai khía cạnh còn lại, gồm khởi đầu và tổ chức-giám sát. Điều này có nghĩa là sinh viên năm nhất chuyên ngành ngôn ngữ Anh tại Đại học Nông Lâm TP. HCM nhận thấy được tầm quan trọng của việc tự học được thể hiện ở ba phương diện cụ thể sau. Thứ nhất là nhóm khởi đầu. Kết quả cho thấy có rất nhiều sinh viên cho rằng việc tiếp cận với giáo viên, bạn bè và truy cập internet để giải đáp thắc mắc giúp họ tự tin và chủ động hơn trong học tập. Ngoài ra, họ còn nhận thấy rằng việc xác định mục tiêu học tập giúp họ định hướng được việc học tốt hơn. Thứ hai là nhóm tổ chức-giám sát. Tương tự với nhóm đầu tiên, các đối tượng khảo sát cũng thừa nhận ảnh hưởng tích cực của việc chọn các hoạt động luyện tập tiếng Anh ngoài lớp học, chọn phương pháp học tập, chiến lược học, xây dựng và thực hiện kế hoạch học tập, tài liệu học trong và ngoài lớp học đối với động lực học của họ. Cuối cùng là nhóm đánh giá. Không giống như hai nhóm trên, nhận thức của sinh viên về các ý kiến trong nhóm được chia thành hai nhóm nhỏ. Nếu như đa số sinh viên nghĩ rằng họ cảm thấy tự tin hơn khi theo dõi tiến trình học tập và có thể xác định được điểm mạnh, điểm yếu khi làm việc với bạn bè thì nhiều trong số họ chưa thật sự tin rằng việc tự đánh giá năng lực của mình giúp đạt kết quả cao hơn và việc suy ngẫm lại hoặc viết nhật ký những gì đã học giúp họ kiểm soát việc học tốt hơn. 


\subsection{Khuyến nghị}

Với kết quả thu được từ nghiên cứu, chúng tôi đề xuất một vài khuyến nghị liên quan đến ba khía cạnh của tự học như sau:

Đối với nhóm khởi đầu, giáo viên cần nêu rõ tầm quan trọng của việc tự học và tính hữu dụng của mục tiêu học tập ngay đầu khóa học để họ có thể xác định những mục tiêu dài hạn và ngắn hạn cho cả khóa học. Ngoài ra, giáo viên cần tạo nhiều cơ hội để tăng tính chủ động, sáng tạo cho sinh viên và các kỹ năng mềm cho sinh viên bằng cách sử dụng phương pháp dạy học theo dự án (project-based) hay nhiệm vụ (task-based). Ví dụ, sau khi hoàn thành bài học về "môi trường”, giáo viên yêu cầu sinh viên làm nhóm, cặp hoặc cá nhân (tùy vào độ khó của bài) để làm một tờ báo tường (poster) về nguyên nhân gây ô nhiễm môi trường hoặc giải pháp cải thiện ô nhiễm môi trường. Với nhiệm vụ này, sinh viên cần làm việc với nhau, tìm kiếm thông tin trực tuyến hoặc các nguồn tài liệu ngoại tuyến để hoàn thành dự án của mình. Từ những hoạt động này, sinh viên sẽ trở nên tích cực hơn trong học tập và dần thay đổi tư duy về trách nhệm của mình đối với việc học.

Đối với nhóm tổ chức-giám sát, giáo viên cần hướng dẫn sinh viên cách xây dựng kế hoạch học tập, chọn phương pháp học, chiến lược học, tài liệu học tập và hoạt động luyện tập ngoài lớp học. Nói cách khác, sinh viên cần kiến thức về những hoạt động này để họ tự tin hơn khi áp dụng. Cụ thể là giáo viên cần chuẩn bị các biểu mẫu hoặc tài liệu cần thiết để giới thiệu và hướng dẫn cho sinh viên cách thực hiện vào buổi đầu tiên của khóa học. Ngoài việc chuẩn bị kiến thức cho sinh viên về các hoạt động tự học, giáo viên cần đề xuất với các cấp lãnh đạo về việc tổ chức các hoạt động ngoài giờ học để thu hút sinh viên tham gia như là câu lạc bộ tiếng Anh, cuộc thi hùng biện tiếng Anh, cuộc thi hát tiếng Anh, cuộc thi tìm hiểu văn hóa nước ngoài, cuộ thi báo tường bằng tiếng Anh, câu lạc bộ dịch thuật, v.v. Bên cạnh việc đưa ra các nguồn học cơ bản, giáo viên cần giới thiệu cho sinh viên nguồn học liệu trực tuyến của trường (nếu có).

Đối với nhóm đánh giá, việc để sinh viên tự đánh giá năng lực của mình hay đánh giá cho bạn cùng lớp là một trong những kỹ năng tự học cần thiết, song trong bối cảnh hiện tại sinh viên chưa được hướng dẫn cách làm cụ thể cho từng môn học nên có phần hoài nghi tính hiệu quả về hoạt động này. Vì thế, giáo viên cần đưa ra thang chấm điểm và tiêu chí rõ ràng để sinh viên có thể tham chiếu và cảm thấy tự tin hơn khi đánh giá. Ngoài ra, giáo viên nên khuyến khích sinh viên suy ngẫm lại những gì mình đã học bằng nhiều hình thức khác nhau như là đặt một vài câu hỏi ngắn sau khi kết thúc bài học, trả lời các câu hỏi cuối bài (nếu có), viết nhật ký, thảo luận cuối giờ, sử dụng trò chơi 3-2-1, etc.

\subsection{Hạn chế và huớng nghiên cứu tiếp theo}

Mặc dù nghiên cứu này ít nhiều mang lại lợi ích cho các nhà nghiên cứu sau này khi họ tìm hiểu về vấn đề tự học nhưng nó cũng tồn tại một số hạn chế sau.

Vì thời gian có hạn nên nghiên cứu này chỉ là sử dụng một công cụ nghiên cứu, đó là bảng khảo sát để thu thập dữ liệu định lượng. Vì thế, các nghiên cứu tiếp theo cần xem xét sử dụng các công cụ thu thập dữ liệu khác như là phỏng vấn, quan sát lớp, viết nhật ký, etc., để bổ sung cho nhau tăng độ tin cậy cho kết quả của bài nghiên cứu. 
Đối tượng khảo sát chỉ giới hạn là sinh viên năm nhất chuyên ngành Ngôn ngữ Anh tại Trường Đại học Nông Lâm Thành phố Hồ Chí Minh. Các nghiên cứu sau này nên so sánh, đối chiếu nhận thức của sinh viên ở các năm để có sự để tìm ra sự giống và khác nhau; từ đó đưa ra những đề xuất phát huy năng lực tự học phù hợp cho sinh viên từng năm.

Các nhà nghiên cứu trong tương lai nên thực hiện nghiên cứu thực nghiệm khi áp dụng một phương pháp mới như là ứng dụng công nghệ thông tin vào việc giảng dạy, phương pháp giảng dạy theo dự án hay nhiệm vụ, v.v. nhằm nâng cao năng lực tự học của sinh viên.

\section{Tài liệu tham khảo}

Aliweh, A. M. (2011). The effect of electronic portfolios on promoting Egyptian EFL college students' writing competence and autonomy. Asian EFL Journal, 13(2), 90-133.

Ban chấp hành Trung ương. (2013). Nghi quyết số 29-NQ/TW ngày 4/11/2013 Hội nghi Trung ương 8 khóa XI về đổi mới căn bản, toàn diện giáo dục và đào tạo [Resolution No. 29NQ / TW dated November 4, 2013 Resolution of the 8th session of the XI Central Conference on fundamental and comprehensive innovation of education and training]. Retrieved January 12, 2020, from https://moet.gov.vn/tintuc/Pages/doi-moi-can-bantoan-dien-gd-va-dt.aspx?ItemID=3928

Benson, P. (2001). Teaching and researching autonomy in language learning. London, England: Longman.

Blidi, S. (2017). Collaborative learner autonomy: A mode of learner autonomy development. Singapore: Springer.

Büyükduman, İ., \& Şirin, S. (2010). Learning Portfolio (LP) to enhance constructivism and student autonomy. Procedia Social and Behavioral Sciences, 3, 55-61. doi:10.1016/j.sbspro.2010.07.012

Chau, J., \& Cheng, G. (2010). Towards understanding the potential of e-portfolios for independent learning: A qualitative study. Australasian Journal of Educational Technology, 26(7), 932-950. doi:10.14742/ajet.1026

Chen, H. I., \& Pan, H. H. (2015). Learner autonomy and the use of language learning strategies in a Taiwanese junior high school. Journal of Studies in Education, 5(1), 52-64. doi:10.5296/jse.v5i1.6972

Cotterall, S. (2000). Promoting learner autonomy through the curriculum: Principles for designing language courses. ELT Journal, 54(2), 109-117. doi:10.1093/elt/54.2.109

Crabbe, D. (1993). Fostering autonomy from within the classroom: The teacher's responsibility. System, 21(4), 443-452.

Dam, L., Eriksson, R., Little, D., Miliander, J., \& Trebbi, T. (1990). Towards a definition of autonomy. In T. Trebbi (Ed.), Third Nordic workshop on developing autonomous learning in the FL classroom (pp. 102-103). Bergen, Norway: University of Bergen.

Dang, T. T. (2012). Learner autonomy perception and performance: A study on Vietnamese students in online and offline learning environments. (Unpublished doctoral dissertation). La Trobe University, Melbourne, Australia. 
Dickinson, L. (1987). Self-instruction in language learning. Cambridge, UK: Cambridge University Press.

Duong, M. T. (2015). A portfolio-based learner autonomy development model in an EFL writing course. (Unpublished doctoral dissertation). Suranaree University of Technology. Nakhon Ratchasima, Thailand.

Duong, M. T., \& Seepho, S. (2014). Promoting learner autonomy: A qualitative study on EFL teachers' perceptions and their teaching practices. Proceedings of the International Conference: DRAL 2 / ILA 2014, 129-137.

Ganza, W. L. (2008). Learner autonomy - Teacher autonomy. In T. Lamb \& H. Reinders (Eds.), Learner and teacher autonomy: Concepts, realities and responses (pp. 63-79). Amsterdam, Netherlands: John Benjamins.

Gardner, D., \& Miller, L. (1999). Establishing self-access: From theory to practice. Cambridge, UK: Cambridge University Press.

George, D., \& Mallery, P. (2003). SPSS for Windows step by step: A simple guide and reference. Boston, MA: Allyn \& Bacon.

Haseborg, H. E. T. (2012). Principles of learner autonomy in action: Effects and perceptionsin a college-level foreign language class. (Doctoral dissertation, College of Education and Human Services, Morgantown, WV). Retrieved December 01, 2019, from https://researchrepository.wvu.edu/etd/3577/

Holec, H. (1981). Autonomy and foreign language learning. Oxford, UK: Pergamon Press.

Ismail, N., \& Yusof, M. A. M. (2012). Using language learning contracts as a strategy to promote learner autonomy among ESL learners. Procedia - Social and Behavioral Sciences, 66, 472-480.

Kurtz, L. M. (2012). Learning from twenty-first century second language learners: A case study in smartphone use of language learners (Master's thesis, Iowa State University, Ames, Iowa). Retrieved December 02, 2019, from https://lib.dr.iastate.edu/etd/12669

Little, D. (1991). Learner autonomy: Definitions, issues and problems. Dublin, Ireland: Authentik.

Little, D. (2004). Learner autonomy, teacher autonomy and the European language portfolio. Retrieved December 02, 2019, from http://utc.fr/ untele/2004ppt/handouts/little.pdf

Little, D. (2009). Language learner autonomy and the European language protfolio: Two L2 English examples. Language Teaching, 42(2), 222-233.

Littlemore, J. (2001). Learner autonomy, self-instruction and new technologes in language learning: Current theory and practice in higher education. In A. Chambers \& G. Davies (Eds.), ICT and language learning: An Enropean perspective (pp. 39-52). Lisse, Netherlands: Swets \& Zeitlinger Publishers.

Littlewood, W. (1997). Design and adapting materials to encourage learner autonomy. In P. Benson \& P. Voller (Eds.), Autonomy \& independence in language learning (pp. 79-91). New York, NY: Longman. 
Liu, H. J. (2012). Understanding EFL undergraduate anxiety in relation to motivation, autonomy, and language proficiency. Electronic Journal of Foreign Language Teaching, 9(1), 123-139.

Macaro, E. (1997). Target language, collaborative learning, and autonomy. Clevedon, UK: Multilingual Matters.

Macià, E. A. I., Ramos, C. R., Cervera, A. S., \& Fuentes, C. B. (2003). Developing learner autonomy through a virtual EAP course at university. Retrieved December 3, 2019, from http://www.publicacions.ub.edu/revistes/bells12/PDF/art01.pdf

Najeeb, S. S. (2013). Learner autonomy in language learning. Procedia - Social and Behavioral Sciences, 70, 1238-1242.

Noytim, U. (2006). The impact of the internet on English languag teaching: A case study at a Thai Rajabhat University. (Doctoral dissertation, University of Technology, Sydney, Australia). Retrieved December 04, 2019, from https://opus.lib.uts.edu.au/bitstream/2100/384/2/02whole.pdf

Nunan, D. (1996). What's my style? In D. Gardner \& L. Miller (Eds.), Tasks for independent language learning (pp. 5-6). Alexandria, Egypt: Teachers of English to Speakers of Other Languages, Inc.

Nunan, D. (1997). Design and adapting materials to encourage learner autonomy. In P. Benson \& P. Voller (Eds.), Autonomy \& independence in language learning (pp. 192-203). New York, NY: Longman.

Nunan, D., \& Lamb, C. (1996). The self-directed teacher: Managing the learning process. Cambridge, UK: Cambridge University Press.

Reid, J. M. (1993). Teaching ESL writing. Englewood Cliffs, NJ: Prentice Hall Regents.

Rivers, W. P., \& Golonka, E. M. (2009). Third language acquisition theory and practice. In M. H. Long \& C. J. Doughty (Eds.), The handbook of language teaching (pp. 250-266). Oxford, UK: Blackwell Publishing.

Rubin, J., \& Thompson, I. (1994). How to be a more successful language learner. Boston, MA: Heinle \& Heinle Publishers.

Sanprasert, N. (2010). The application of a course management system to enhance autonomy in learning English as a foreign language. ScienceDirect, 38, 109-123.

Scharle, Á., \& Szabó, A. (2000). Learner autonomy: A guide to developing learner responsibility. Cambridge, UK: Cambridge University Press.

Scheb-Buenner, P. (2018). University students' perception on autonomous learning: A case of private university, Thailand. In Proceedings of The 1st International Conference on English Studies Organizing Committee (pp. 321-332). Krabi, Thailand: Thaksin University.

Stevens, R. J. (2007). Cooperative learning and literacy instruction in middle level education. In R. M. Gillies, A. Ashman, \& J. Terwel (Eds.), The teacher's role in implementing cooperative learning in the classroom (pp. 92-109). New York, NY: Springer. 
Thanasoulas, D. (2000). What is learner autonomy and how can it be fostered? The Internet TESL Journal, 6(11). Retrieved December 04, 2020, from http://iteslj.org/Articles/Thanasoulas-Autonomy.html

Voller, P. (1997). Does the teacher have a role in autonomous language learning? In P. Benson P. Voller (Eds.), Autonomy \& independence in language learning (pp. 98-113). London, UK: Longman.

Wenden, A. (1991). Learner strategies for learner autonomy: Planning and implementing learner training for language learners. New York, NY: Prentice Hall.

Yunus, M. M., \& Arshad, N. D. M. (2015). ESL teachers' perceptions toward the practices and prospects of autonomous language learning. Asian Social Science, 11(2), 41-51. 